\title{
El caso del barrio la Isla de Villavicencio, Meta - Colombia: la inseguridad y su sistema social
}

\section{The case of the neighborhood of Villavicencio La Isla, Meta - Colombia: insecurity and its social system}

\author{
CASTRO-GARZÓN, Hernando ${ }^{1}$ \\ PUYO, Leidy N. ${ }^{2}$ \\ RODRIGUEZ, Juan P. ${ }^{3}$
}

\begin{abstract}
Resumen
El presente artículo aborda la sociedad como la máxima expresión del sistema social; para el caso de estudio la inseguridad y sus variables transformadoras de la dinámica y desarrollo del barrio La Isla en la ciudad de Villavicencio, Meta, Colombia. Se aplicó un método de triangulación de datos con la variable de inseguridad para caracterizar el sistema social, definir su historia, acceso a la educación, la delincuencia la drogadicción y su ingerencia en la dinámica familiar.

Palabras clave: sostenibilidad, sociedad, comunidad.

Abstract

This article addresses society as the ultimate expression of the social system; for the case study the insecurity and its transformative variables of the dynamics and development of the neighborhood La Isla in the city of Villavicencio, Colombia. A data triangulation method was applied with the variable of insecurity to characterize the social system, define its history, access to education, crime drug addiction and its ingerence in family dynamics.

key words: sustainability, society, community.
\end{abstract}

\section{Introducción}

Como base para el estudio se partio de la teoría de los sistemas, explica que "la capacidad de los procesos y fenómenos existentes de entablar interacciones de tal naturaleza que como resultado de las mismas, forman sistemas" (Jimenez, 2017, pág. 35), tomando como referente de estudio el sistema social descrito por (Achong, 2015, pág. 2) “como el resultado de la co-evolución entre la naturaleza y la sociedad.(...)"; en donde existe la necesidad intrínseca del equilibrio y colaboración de sus componentes, para conseguir un desarrollo sostenible explican (Arnold \& Rodríguez, 1999); sin embargo dichas relaciones pueden ser interrumpidas o modificadas por las condiciones con las que el sistema interactúa, como el entorno geográfico y físico, la constitución espacial, los sistemas aledaños, entre otros; por tanto, dando lugar a los conflictos sociales como fenómenos que alteran

\footnotetext{
1 Profesor. Facultad de Ciencias Ecónomicas. Universidad de los Llanos, hcastro@unillanos.edu.co

${ }^{2}$ Maestrante. Gestión del desarrollo Sustentable. Universidad de Guerrero, leidy.puyo@unillanos.edu.co

${ }^{3}$ Profesor Titular. Facultad de medio Ambiente y Recursos Naturales. Universidad Distrital Francisco Jóse de Caldas, jprodriguezm@udistrital.edu.co
} 
los comportamientos naturales y equilibrados del sistema, junto con sus componentes fundamentales: la familia y el individuo según (Guerreroet al.,(2016) y (Preister, 1980) en el caso de la sociedad.

Según lo establecido, la inseguridad hace parte de uno de estos conflictos; se entiende como un fenomeno del comportamiento que nace desde el individuo que la inflige y el entorno en que se desarrolla; así como la interacción con las instituciones, la familia, que determinan su nivel socio economico y educativo (Solis Sosa, 2015). Por lo cual este fenomeno es descrito como "una de las principales preocupaciones en las sociedades contemporáneas, que ha ocurrido en distintos momentos y circunstancias del acontecer nacional" (Rojas \& De la Puente Lafoy, 2005, pág. 63), vislumbrandose en conflictos con incidencia política, institucional y armada contra víctimas, sociedad y estado (Duque Gómez, 2017). En donde los agentes adquieren un rol primordial, entendiendo que "la seguridad hace referencia a la satisfacción de los derechos básicos de un individuo en distintos ámbitos" (García Martínez, et. al, 2008).

Ahora bien, en relación al estado del fenómeno en el contexto latinoamericano, para la Comisión Económica para América Latina (CEPAL, 2017) reconoce la inseguridad como un factor concurrente de las problemáticas sociales, posicionándolas como críticas para un continente en donde predomina la desigualdad social y la pobreza. Con respecto a Colombia, el Índice Global de Paz la ubicó en 2017/2018 en el lugar 29 y 27 de los países más riesgosos del mundo, esto por la guerra interna, generando una asociación directa del concepto de inseguridad al conflicto armado así como con narcotráfico , (IEP, 2018); en el departamento del Meta se presenta el mismo fenomeno, por las causales anteriormente anotadas, agravado por el vulneramiento de comunidades, desplazamiento forzado y la violación de los derechos humanos por grupos al margen de la ley (Gobernación del Meta, 2017). A cuenta de la dinámica nacional y departamental la ciudad de Villavicencio ha absorbido los efectos de la violencia, traducidos en inseguridad vinculado al delito común, clasificándola sobre el promedio nacional en hurtos, homicidios y secuestros (DNP, 2017).

El presente trabajo pretende mostrar una caracterización del sistema social, la percepción de la comunidad así como la relación con las autoridades en el barrio La Isla de la ciudad de Villavicencio, estableciendo las relaciones entre estos y los fenómenos de inseguridad presentes.

\section{Metodología}

Para identificar las características de un sistema social vulnerable impactado por la inseguridad, se aplica en el barrio La Isla en la ciudad de Villavicencio, Meta - Colombia, una metodología mixta con un diseño de investigación no experimental de orden transeccional descriptivo, triangulando los datos obtenidos en un censo. Definiendo como variable independiente la inseguridad, ésta relación se da para entender las variables asociadas teóricamente a ella; para lo que se abordo primero un anclaje conceptual sobre la teoría de los sistemas, la inseguridad como fenómeno y las variables de inseguridad definidas por la instituciónalidad del país. La información se obtuvo a través de un instrumento compuesto por preguntas dicotómicas enfocadas en relacionar las variables determinantes de inseguridad en el barrio y otras de carácter abierto, para establecer la percepción y confianza de la comunidad en las instituciones frente a los grupos delincuenciales.

\section{Resultados}

El barrio La Isla fue reconocido por el Decreto 084 de 1992 donde se crea y determina lindero; establecido como parte de la comuna dos de Villavicencio, Meta Colombia y asignado con estratificación económica 2, participó en la fundación de los primeros asentamientos de la ciudad y al igual que los barrios aledaños inicio como una invasión (Motta, 2015). Imagen 1. 
Imagen 1

Delimitación del barrio La Isla.

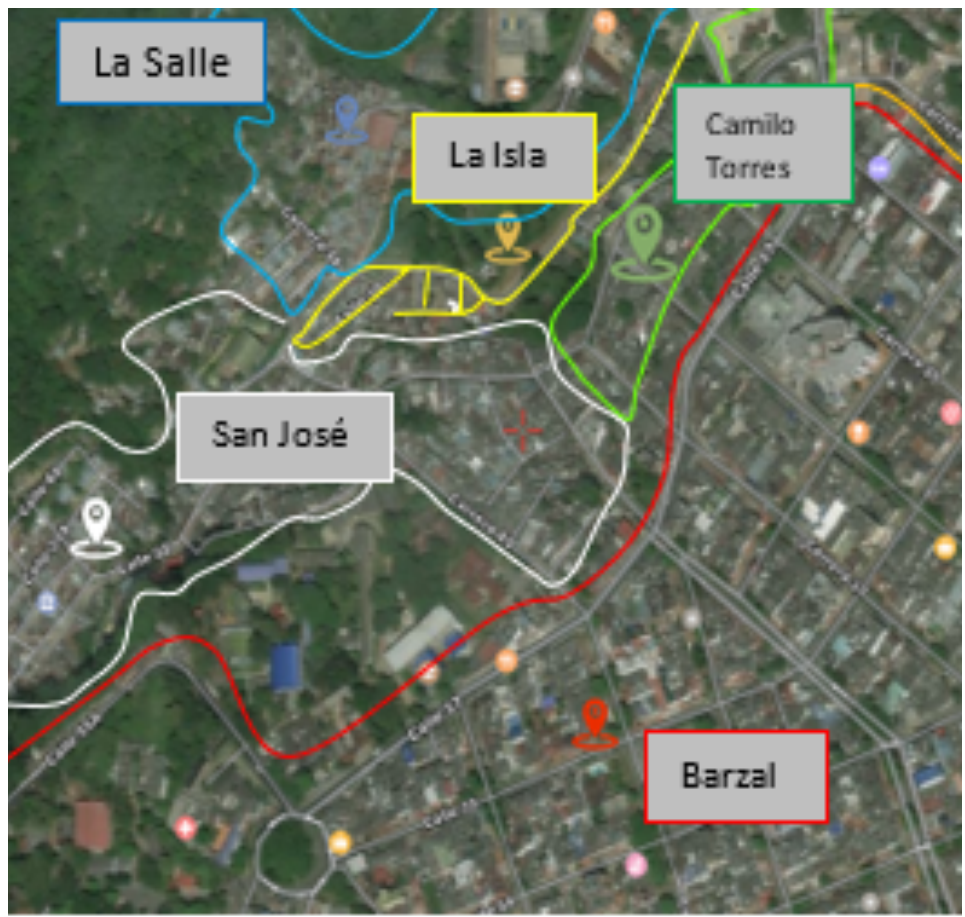

Fuente: Google Maps (2020) modificado por los autores

Para la identificación y caracterización de la población del barrio se establece un censo con la colaboración de la la junta de acción comunal para identificar el número de familias, habitantes y estudiantes por vivienda. Tabla 1. La población habitante de la zona es de 180 personas incluyendo niños y adultos, con un total de 84 familias que habitan en 45 viviendas, evidenciando un alto numero de personas por casa, con una media de al menos 2 familias; sin embargo se evidenció concentración en las que tienen una disposición aislada, presentándose en las de más de una planta una relación proporcional con las que habitan más de una.

Tabla 1

Censo barrio La isla.

\begin{tabular}{|c|c|c|c|c|c|c|c|}
\hline Casa $\mathbf{N}^{\circ}$ & $\mathbf{N}^{\circ}$ familias & Habitantes & Estudiantes & Casa $\mathbf{N}^{\circ}$ & $\mathbf{N}^{\circ}$ familias & Habitantes & Estudiantes \\
\hline 1 & 1 & 6 & 1 & 28 & & & \\
\hline 2 & 2 & 4 & 1 & 29 & 1 & 2 & \\
\hline 3 & 2 & 4 & 1 & & & & \\
\hline 4 & 4 & 7 & 1 & 30 & 1 & 3 & 1 \\
\hline 5 & 2 & 6 & 1 & 31 & 2 & 3 & 1 \\
\hline 6 & 3 & 4 & 1 & 32 & 1 & 2 & \\
\hline 7 & 3 & 4 & 1 & 33 & 1 & 2 & \\
\hline 8 & 2 & 4 & 1 & 34 & 1 & 3 & 1 \\
\hline 9 & 1 & 3 & 1 & 35 & & & \\
\hline 10 & 3 & 3 & 1 & 36 & 1 & 3 & \\
\hline 11 & & & & 37 & 1 & 4 & \\
\hline 12 & 3 & 10 & 1 & 38 & 1 & 4 & 1 \\
\hline 13 & 1 & 2 & 1 & 39 & 1 & 2 & \\
\hline 14 & 2 & 4 & 1 & 40 & 1 & 2 & \\
\hline 15 & 4 & 15 & 1 & 41 & 1 & 3 & 1 \\
\hline 16 & 1 & 2 & & 42 & 1 & 2 & \\
\hline
\end{tabular}




\begin{tabular}{|c|c|c|c|c|c|c|c|}
\hline Casa $\mathbf{N}^{\circ}$ & $\mathbf{N}^{\circ}$ familias & Habitantes & Estudiantes & Casa $\mathbf{N}^{\circ}$ & $\mathbf{N}^{\circ}$ familias & Habitantes & Estudiantes \\
\hline 17 & 4 & 5 & 1 & 43 & 2 & 4 & 1 \\
\hline 18 & 1 & 3 & 1 & 44 & 1 & 3 & \\
\hline 19 & 2 & 3 & 1 & 45 & 1 & 3 & 1 \\
\hline 20 & 6 & 15 & 1 & 46 & 1 & 2 & 1 \\
\hline 21 & 1 & 2 & & 47 & & & \\
\hline 22 & 6 & 6 & 1 & 48 & 1 & 3 & 1 \\
\hline 23 & 1 & 2 & 1 & 49 & 1 & 3 & 1 \\
\hline 24 & & & & 50 & 1 & 2 & \\
\hline 25 & 4 & 5 & & 51 & & & \\
\hline 26 & & & & 52 & 1 & 4 & 1 \\
\hline \multirow[t]{2}{*}{27} & 2 & 2 & & 53 & & & \\
\hline & & & & TOTAL & 84 & 180 & 31 \\
\hline
\end{tabular}

Fuente: Los autores (2019)

Se consideró el estado de las viviendas (número de pisos evidentes), las familias habitantes y los establecimientos comerciales que se pudieran encontrar. Tabla 2. El 28\% de las casa son de una planta; la cuarta parte de los predios del barrio cubren las condiciones básicas de servicios públicos para los requerimientos de una familia, sin embargo es importante considerar que en el $44 \%$ habita más de una; existiendo relación alta entre la presencia de varias familias por hogar y el número de viviendas con más de una planta; adicionalmente, referente a los establecimientos comerciales están presentes en un $9 \%$ de las casas, observándose a pesar de las características de la zona que se ha promovido el emprendimiento formal e informal.

Tabla 2

Situación de vivienda y comercio.

\begin{tabular}{lllll}
\hline Numero de casas & \multicolumn{2}{l}{$\begin{array}{l}\text { En el censo se encuentran } 53 \text { casas, } 14 \text { casas de dos } \\
\text { pisos y 1ä de tres pisos, restantes de } 1 \text { piso. }\end{array}$} \\
\hline $\begin{array}{l}\text { Numero de establecimientos de } \\
\text { comercio }\end{array}$ & $\begin{array}{l}4 \text { establecimientos (tiendas de barrio) y } \\
\text { parqueadero }\end{array}$ \\
\hline Numero de casas encuestadas & Se consiguen encuestar 45 casas & \\
\hline
\end{tabular}

Fuente: Los autores (2019)

A continuación se presenta lo relacionado al componente social presente en el barrio La Isla. Tabla 3. En cuanto a la educación, en el $58 \%$ de las viviendas se relacionarón estudiantes de diferentes niveles, en consideración al $69 \%$ en las que se encuentran individuos en edad de acceder a educación básica. El factor emprendimiento es del $28 \%$, significante con respecto al tamaño del barrio, considerando que solo el $44 \%$ de la comunidad tiene un ingreso mayor al salario mínimo legal; agregando que los integrantes más jóvenes de las familia presentan un nivel de ocupación bajo. 
Tabla 3

Componentes del sistema social.

\begin{tabular}{|c|c|c|c|c|c|}
\hline \multicolumn{2}{|c|}{$\begin{array}{l}\text { Relacional } \\
\text { Población }\end{array}$} & \multicolumn{4}{|c|}{$\begin{array}{l}84 \text { familias y } 180 \text { individuos en } 45 \text { casas; } 31 \text { familias con menores en edad de estudiar, } 44 \% \text { de } \\
\text { las viviendas habita mas de una familia. Índice de población desplazada } 49 \% \text {. }\end{array}$} \\
\hline \multirow[t]{3}{*}{$\begin{array}{c}\text { Organización } \\
\text { social } \\
\text { Cultura } \\
\text { Historia }\end{array}$} & Recursos & $\begin{array}{l}\text { Primeros asentamientos de } \\
\text { Villavicencio, Invasión-> } \\
\text { barrio lindero (Decreto } \\
084 / 1992 \text { ). } \\
\text { 43\% familias tiene ingresos } \\
\text { superiores al SMLV, } 62 \% \text { de } \\
\text { las familias con al menos un } \\
\text { integrante desempleado. }\end{array}$ & $\begin{array}{l}\text { Relaciones } \\
\text { regulativas }\end{array}$ & Economía & $\begin{array}{l}5 \text { establecimientos } \\
\text { de comercio, } \\
\text { organización } \\
\text { económica } \\
\text { delincuencial (micro } \\
\text { extorsión, expendio } \\
\text { de drogas, ventas e } \\
\text { intercambio de } \\
\text { artículos robados), } \\
\text { factor de } \\
\text { emprendimiento } 28 \%\end{array}$ \\
\hline & Servicios & $\begin{array}{l}\text { 93\% de las viviendas cuenta } \\
\text { con los servicios básicos, } \\
\text { difícil acceso de redes de } \\
\text { internet. }\end{array}$ & & $\begin{array}{l}\text { Organización } \\
\text { administrativa }\end{array}$ & $\begin{array}{c}\text { Barrio lindero; de la } \\
\text { carrera } 34 \text { con calle } \\
38, \\
\text { abarcando en la calle } \\
38 \text { las carreras } 36, \\
37,38,38 \text { A y la Calle } \\
39 \text { y } 39 \text { A; } 54 \\
\text { viviendas ( } 14 \text { de } 2 \\
\text { plantas, } 1 \text { de } 3 \text { ). } \\
\text { Cuenta con junta de } \\
\text { acción comunal. }\end{array}$ \\
\hline & Educación & $\begin{array}{l}58 \% \text { de las viviendas tienen } \\
\text { estudiantes vs } 69 \% \text { viviendas } \\
\text { con individuos en edad de } \\
\text { estudiar }\end{array}$ & & $\begin{array}{l}\text { Relación } \\
\text { internacional }\end{array}$ & $\begin{array}{c}96 \% \text { percibe } \\
\text { presencia policial, } \\
\text { poca confianza en la } \\
\text { policía y entidades } \\
\text { del gobierno,76\% se } \\
\text { sienten expuestos a } \\
\text { ser victimas de } \\
\text { delitos. }\end{array}$ \\
\hline
\end{tabular}

Fuente: Los autores (2019)

Por otro lado, la relación de la población con la institucionalidad es a través de la Policía Nacional, se encuentra que el 96\% percibe su presencia continua; frente a la eficiencia, el $80 \%$ de la población dice acudir a la policía en caso de ser necesario. En contraste, la exposición a delitos resulto ser de un $76 \%$ y el conocimiento de la comunidad sobre quiénes son los delincuentes que participan de forma activa es del 69\%; así como la posibilidad de ser víctimas por parte de las organizaciones criminales, ya que el $76 \%$ de los encuestados teme ser afectado de algún acto violento dentro del barrio incluyendo micro extorsión y agresiones. Ahora bien, el porcentaje de víctimas de violencia por delincuencia común es del $22 \%$, testigos de micro extorsión $22 \%$ y de consumo de drogas el $18 \%$ de la población encuestada. Figura 1.

Ahora bien, sobre la eficiencia de las autoridades se obtuvo que el $69 \%$ de la población encuestada conoce a los delincuentes en la zona, aún conservan temor a ser víctimas de atraco $22 \%$ y otros crímenes $76 \%$, pero no denuncian ante las autoridades. Por otro lado el conocimiento de la comunidad que los delincuentes son consumidores de sustancias psicoactivas es del $87 \%$, en contraste, se observa que la tasa de consumo para la población estudiada es del 18\% . Figura 2, 3 y 4. 
Figura1

Relación presencia policial delitos

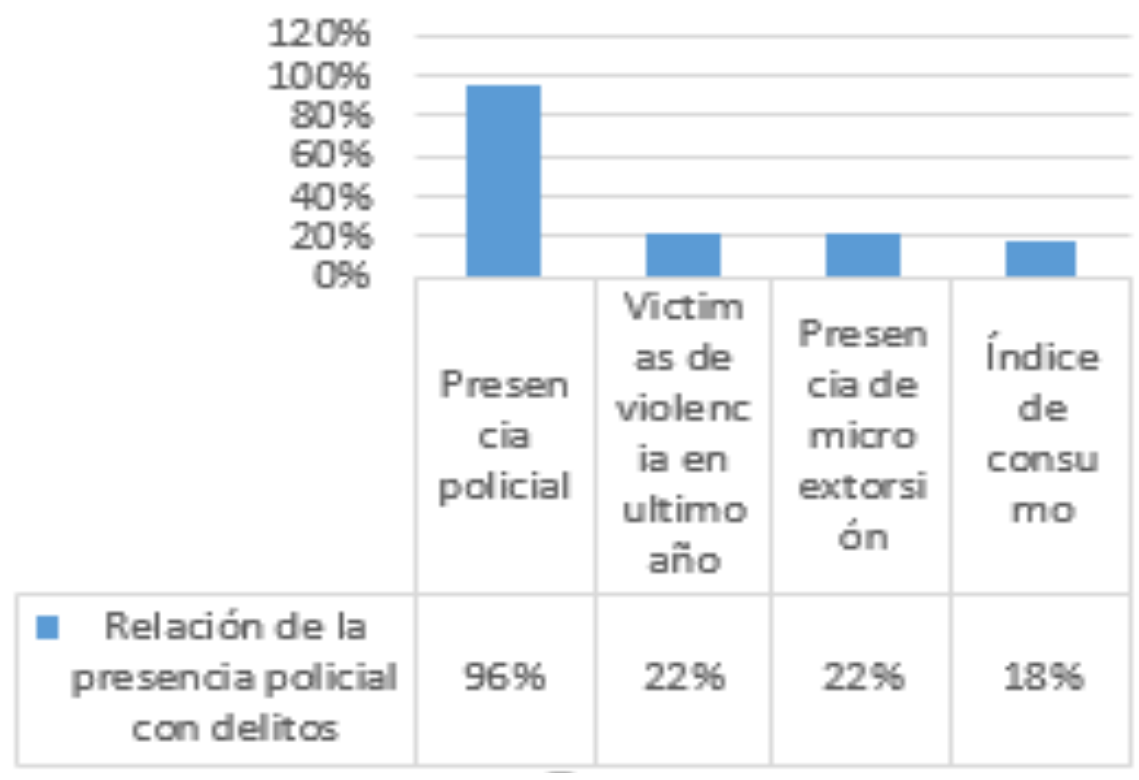

Fuente: los autores (2019)

Figura 2

Relación eficiencia policías vs conocimiento de delitos por parte de la comunidad

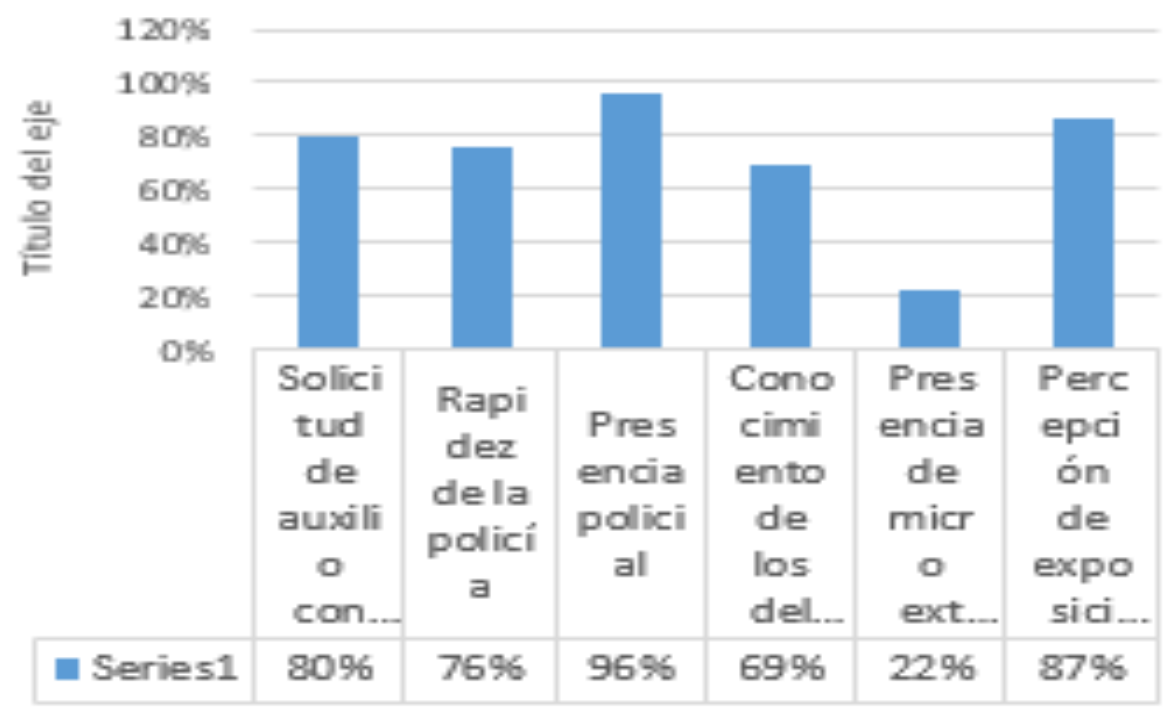

Fuente. Los autores (2019) 
Figura 3

Relación confianza en las autoridades vs eficiencia

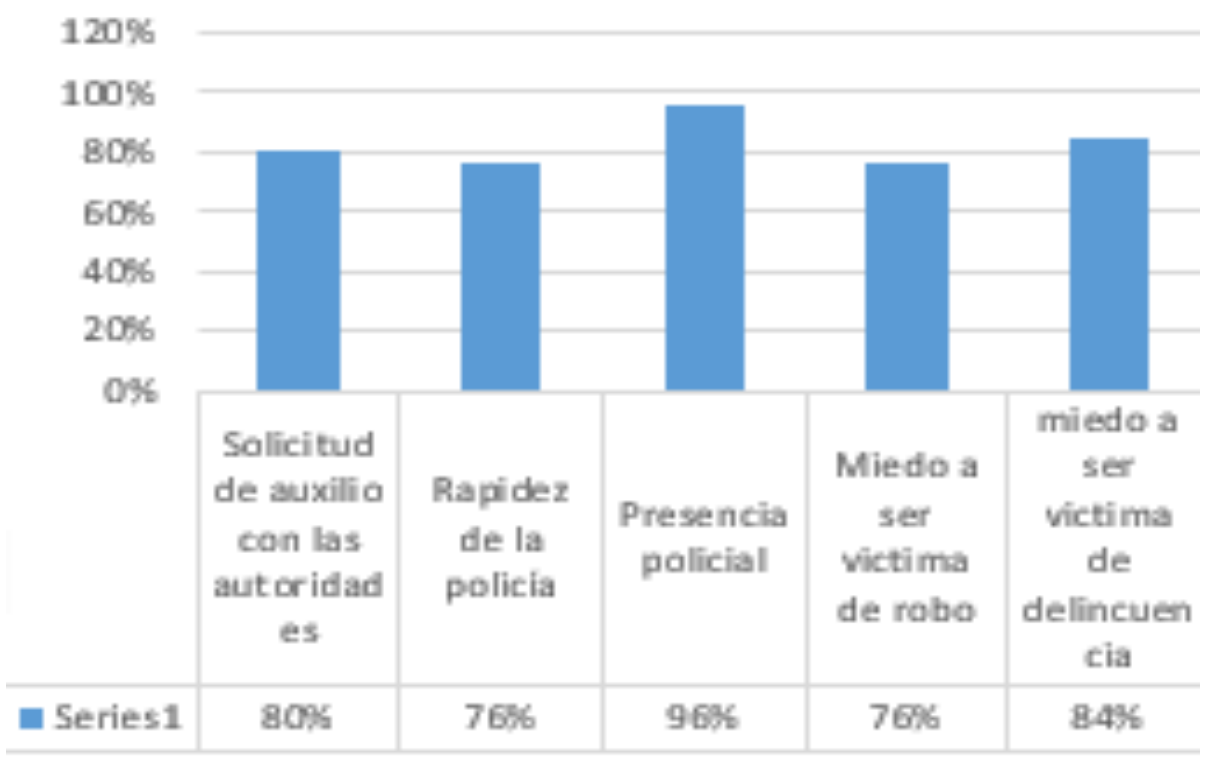

Fuente. Los autores (2019)

Figura 4

Interacción de la comunidad con la delincuencia.

\section{$100 \%$}

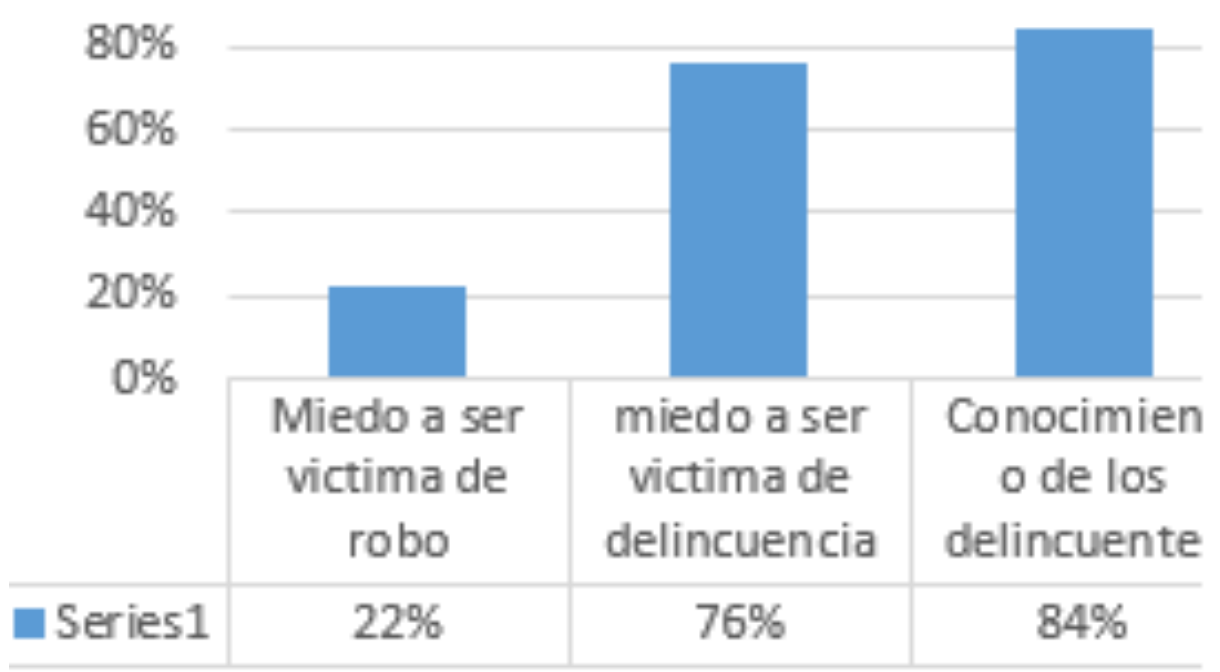

Fuente. Los autores (2019)

\section{Conclusiones}

La población presente se tipifica en familias numerosas víctimas de desplazamiento forzado y presencia de desempleo; como característica prevaleciente el ingreso es superior al salario mínimo legal en los sujetos económicamente activos, ya que varios individuos con ocupación conforman el núcleo familiar. Se constato que el nivel de ingresos y la ocupación influyen directamente en el estrés de los hogares, por tanto en la violencia 
intrafamiliar; concluyendo que los conflictos se generan principalmente por problemas de ingresos, desempleo y acceso a la educación.

Se identificó que la población desplazada por la violencia, asentada en la zona de estudio, no cuenta en su mayoría con empleos formales con una alta vulnerabilidad hacia la delincuencia presente. Las variantes más significantes en el sistema social del barrio con respecto de la dinámica familiar son: la pobreza, el desempleo, la violencia intrafamiliar y la violación de los derechos humanos. El joven de la comunidad se ve muy influenciado por el medio (organismo social, sistemas conjuntos), el entorno (entidades con que se relaciona) además del contexto (constitución espacial) que trae como consecuencia un acercamiento al delito, por la facilidad de interacción con las organizaciones delictivas, lo que especialmente en los menores implica la transformación de su modelo de vida como los ejemplos y figuras a seguir.

Frente al reconocimiento de las organizaciones delictivas y los individuos que participan en ellas por parte de la comunidad analizada, se relaciona que a pesar de una alta presencia de la policía y la rapidez de respuestas no representa un impacto frente a la frecuencia de delitos en la zona, la exposición al consumo de sustancias psicoactivas y de menores que se involucren en esa dinámica del sector; determinando que las estrategias implementadas por la institución no están siendo efectivas.

El fenómeno de la inseguridad como problemática social es un transformador de la dinámica familiar y del desarrollo del individuo dentro de la misma, dado que los mas jóvenes observan comportamientos y estructuras económicas delincuesiales que son un modelo a seguir o que quieren emular generando un efecto bola de nieve y permanente en la inseguridad creciente de la ciudad.

\section{Referencias bibliográficas}

Achong, J. C. (2015). Centro Ideas. Recuperado el 26 de 05 de 2018, de Socioecosistema: un concepto necesario para la investigación y la gestión del desarrollo sustentable.:

http://ideas.org.pe/index.php/videos/item/545-socioecosistema-un-concepto-necesario-para-lainvestigacion-y-la-gestion-del-desarrollo-sustentable.

Arnold, C. M., \& Rodríguez, M. D. (1999). Sociedad y teoría de sistemas : elementos para la comprensión de la teoría de Niklas Luhman (3 ed.). Santiago de Chile, Chile: EDITORIAL UNIVERSITARIA S.A.

CEPAL- Comisión Económica para América Latina y el Caribe, (2017). Panorama Social de América Latina 2016. Obtenido de Informes anuales: https://www.cepal.org/es/publicaciones/41598-panorama-social-americalatina-2016

DNP. (2017). Panorama regional "pobreza monetaria y multidimensional, panorama departamental: necesidad de politicas publicas diferenciales". 3. Colombia.

https://colaboracion.dnp.gov.co/CDT/Desarrollo\%20Territorial/Portal\%20Territorial/KitSeguimiento/Pobr eza/Publicaci\%C3\%B3n\%20lpm\%20deptal.pdf

Duque Gómez, L. F. (2017). Conflicto social colombiano: representación en textos escolares de ciencias sociales. 9(19), 20. Pereira, Colombia. doi:https://doi.org/10.11144/Javeriana.m9-19.cscr

García Martínez, C., Panadero Moya, M., \& De León Herrera, R. (2008). MANIFESTACIONES DE LA POBREZA EN CARTAGENA DE INDIAS (COLOMBIA). En U. d. Barcelona (Ed.), X Coloquio Internacional de Geocrítica: DIEZ AÑOS DE CAMBIOS EN EL MUNDO, EN LA GEOGRAFÍA Y EN LAS CIENCIAS SOCIALES, 1999-2008. Barcelona. Recuperado el 12 de 03 de 2019, de http://www.ub.edu/geocrit/-xcol/284.htm 
Gobernación del Meta. (2017). Gobernación del Meta. "LAS VÍCTIMAS, SUJETOS DE ESPECIAL ATENCIÓN EN EL POSTCONFLICTO: https://intranet.meta.gov.co/secciones_archivos/813-38569.pdf

Guerrero Escamilla, J. B., Franco Sánchez, L. M., \& Bass Zavala, S. (2016). El sistema de cohesión social y sus efectos en la tasa de delincuencia. CIENCIA ergo-sum: revista científica multidisciplinaria de la Universidad Autónoma del Estado de México, 23(1), 5-16.Institute for Economic \& Peace. (2017). Global Terrorism Index 2017. Recuperado el 01 de 05 de 2018, de http://visionofhumanity.org/app/uploads/2017/11/Global-Terrorism-Index-2017.pdf

IEP- Institute for Economic \& Peace. (2018). Global Terrorism Index 2018. Recuperado el 22 de 02 de 2019, de http://visionofhumanity.org/app/uploads/2018/12/Global-Terrorism-Index-2018-1.pdf

Jiménez, R. H. (2007). SISTEMA Y LO SISTÉMICO EN EL PENSAMIENTO CONTEMPORÁNEO. Ingenieria, 17(2), 37 52. doi:1409-2441

Preister, S. (1980). La teoria de sistema como marco de referencia para el estudio de la familia. Washintong D.C.: Pontifica Universidad de Washintong D.C. Recuperado el 18 de 08 de 2018, de https://repositorio.uc.cl/bitstream/handle/11534/6267/000380040.pdf?sequence=1

Rojas, E. T., \& De la Puente Lafoy, P. (2005). Seguridad Ciudadana y sistemas sociales autorreferentes en el contexto de la sociedad compleja. Urbe et ius: revista de opinión jurídica, (3), 61-80.

Motta, T. A. (2015). Las 2 Orillas. Gramalote, una historia por contar, de https://www.las2orillas.co/gramaloteuna-historia-por-contar/

Solis Sosa, H. (2015). Universidad Católica Santo Toribio de Mogrovejo. (U. C. Mogrovejo, Editor) Inseguridad ciudadana, problema social.: http://www.usat.edu.pe/articulos/inseguridad-ciudadana-problema-social/

Esta obra está bajo una Licencia Creative Commons Attribución-NoCommercial 4.0 International

(cc) BY-NC 